INPLASY

PROTOCOL

To cite: Gao et al. Acupuncture for the treatment of leptin resistance in obesity: a systematic review and metaanalysis protocol. Inplasy protocol 202150056. doi: 10.37766/inplasy2021.5.0056

Received: 15 May 2021

Published: 16 May 2021

Corresponding author: Mingjun Liu

mingjunliu646590@163.com

Author Affiliation: Changchun University of Chinese Medicine

Support: NSFC (Grant No. 86174092)

Review Stage at time of this submission: The review has not yet started.

Conflicts of interest: None declared.

\section{Acupuncture for the treatment of leptin resistance in obesity: a systematic review and meta-analysis protocol}

Gao, T1; Ma, D2; Chen, S3; Zhang, X4; Han, Y5; Liu, M6.

Review question / Objective: The aim of this study is to investigate the effectiveness of acupuncture in obesity with leptin resistance(LR).

Condition being studied: Recent years have witnessed a global increase in obesity and obesity-related diseases. The prevention and treatment of obesity have become one of the most significant public health challenges worldwide in the 21 st century, and how to effectively curb the occurrence of obesity has become a major global concern. Numerous studies have shown that the majority of obese individuals do not respond to leptin, and instead demonstrate leptin resistance.Clinical studies have found that acupuncture is widely used in the clinical treatment of obesity in recent years, but whether it can improve leptin resistance has not been systematically reviewed.This study aimed to investigate the effectiveness of acupuncture in obesity with leptin resistance(LR).

INPLASY registration number: This protocol was registered with the International Platform of Registered Systematic Review and Meta-Analysis Protocols (INPLASY) on 16 May 2021 and was last updated on 16 May 2021 (registration number INPLASY202150056).

\section{INTRODUCTION}

Review question / Objective: The aim of this study is to investigate the effectiveness of acupuncture in obesity with leptin resistance(LR).

Condition being studied: Recent years have witnessed a global increase in obesity and obesity-related diseases. The prevention and treatment of obesity have become one of the most significant public health challenges worldwide in the 21st century, and how to effectively curb the occurrence of obesity has become a major global concern. Numerous studies have shown that the majority of obese individuals do not respond to leptin, and instead 
demonstrate leptin resistance. Clinical studies have found that acupuncture is widely used in the clinical treatment of obesity in recent years, but whether it can improve leptin resistance has not been systematically reviewed. This study aimed to investigate the effectiveness of acupuncture in obesity with leptin resistance(LR).

\section{METHODS}

Participant or population: According to the diagnostic criteria, all participants should be diagnosed obesity with LR. The age, sex, course, number and ethnicity of participants were not limited.

Intervention: Patients in the experimental groups received needle acupuncture as the main intervention which included manual acupuncture, electro-acupuncture, auricular acupuncture and acupoint injection.

Comparator: The control group was treated with the oral medicine, placebo, sham acupuncture, or lifestyle modification.

Study designs to be included: The type of literature gathered must be based on RCT trials. Do not limit the use of blind methods, but the authors should clearly claim that they performed random grouping.

Eligibility criteria: At least including serum leptin level, BW, body mass index(BMI), Waist-to-Hip Ratio(WHR), any 2 items of effective rate [effective rate $=$ (recovery + significant effect + effective)/total number of cases].

Information sources: Electronic databases: Pubmed, Cochrane Library, Embase databases, China National Knowledge Infrastructure (CNKI), Wan Fang, Technology Journal Database (VIP), China Biology Medicine disc(CBM), Chinese Clinical Trial Registry(ChiCTR). Ask the author for complete information if the artical is incomplete.

Main outcome(s): At least including serum leptin level, BW, body mass index (BMI), Waist-to-Hip Ratio (WHR), any 2 items of effective rate [effective rate=(recovery + significant effect + effective)/total number of cases].

Quality assessment / Risk of bias analysis: Two reviewers will independently assesses the quality of the selected studies according to the Cochrane Collaboration's tool for randomized controlled trials. Items will be evaluated in three categories; Low risk of bias, unclear bias and high risk of bias.The following characteristics will be evaluated: Random sequence generation (selection Bias) Allocation concealment (selection bias) Blinding of participants and personnel (performance bias) Incomplete outcome data (arttrition boas) Selective reporting (reporting bias). Other biases results from these questions will be graphed and assessed using Review Manager5.3.

Strategy of data synthesis: Risk ratio (RR) for both fixed and random effects models(weighting by inverse of variance) will be used.A continuity correction will also be used for cells with zero values.Between-study heterogeneity will be assessed using the $t^{2}, X^{2}$ (Cochran $Q$ ) and $I^{2}$ statistic.According to the Cochrane handbook, the $I^{2}$ will be considered nonimportant(60\%).Results will be assessed using forest plots and presented as RRs for the main outcome and secondary outcomes. An influence analysis will be performed to ascertain the results of the meta-analysis by excluding each of the individual studies. Publication bias will be assessed by a funnel plot for meta-analysis and quatified by the Egger method. Statistical analysis will be conducted using STATA software for Mac v15.0 (Stata Corp., College Station,Texas).

Subgroup analysis: We will consider subgroups such as jurisdiction, clinic type, and location.

Sensitivity analysis: The main methods of sensitivity analysis are: changing the inclusion criteria (especially the controversial studies), excluding lowquality studies, using different statistical 
methods/models to analyze the same data, etc. For example, after excluding a lowquality study, the combined effect amount was re-estimated and compared with the results of Meta-analysis before exclusion to explore the impact of the study on the combined effect amount and the robustness of the results. If there is no significant change in the results after exclusion, the sensitivity is lower, and the results are more robust and credible; on the contrary, if there is a significant difference or even opposite conclusion after exclusion, the sensitivity is higher and the robustness of the results is lower, cARE should be taken in interpreting the results and drawing conclusions, suggesting that there are important and potentially biased factors associated with the effectiveness of the intervention.

\section{Language: English.}

\section{Country(ies) involved: China.}

Keywords: Acupuncture; leptin resistance; obesity; meta-analysis; systematic review.

Contributions of each author:

Author 1 - Tianjiao Gao - The author drafted the manuscript.

Email: 347686079@qq.com

Author 2 - Dehui Ma - The author provided statistical expertise.

Email: 610869137@qq.com

Author 3 - Shaotao Chen - The author contributed to the development of the selection criteria, and the risk of bias assessment strategy.

Email: 55835794@qq.com

Author 4 - Xiaolin Zhang - The author read, provided feedback and approved the final manuscript.

Email: 630159087@qq.com

Author 5 - Yiran Han - The author is a corresponding author and provides project funding support.

Email: 277905221@qq.com

Author 6 - Mingjun Liu - The author is a corresponding author and provides project funding support.

Email: mingjunliu646590@163.com 